\title{
Low molecular weight fucoidan attenuates experimental abdominal aortic aneurysm through interfering the leukocyte-endothelial cells interaction
}

\author{
MIN ZHOU*, YONG DING* , LIANG CAI, YONGGANG WANG, CHANGPO LIN and ZHENYU SHI \\ Department of Vascular Surgery, Zhongshan Hospital, Institute of Vascular Surgery, \\ Fudan University, Shanghai 200032, P.R. China
}

Received November 26, 2017; Accepted March 9, 2018

DOI: $10.3892 / \mathrm{mmr} .2018 .8765$

\begin{abstract}
Low molecular weight fucoidan (LMWF) is a sulfated polysaccharide extracted from Saccharina Japonica that presents high affinity for P-selectin and abolish selectin-dependent recruitment of leukocytes. We hypothesized that dietary intake of LMWF, as a competitive binding agent of P-selectin, could limit the inflammatory infiltration and aneurysmal growth in an Angiotensin II-induced abdominal aortic aneurysm (AAA) mouse model. The Gene Expression Omnibus database was used for gene expressions and gene set enrichment analysis. Kyoto Encyclopedia of Genes and Genomes pathway enrichment analysis showed that focal adhesion was involved in the development of AAA. However, dietary intake of LMWF could limit the enlargement of AAA, decreasing maximal aortic diameter and preserving elastin lamellae. Although LMWF did not decrease the circulatory monocytes count and lower the expression of P-selectin in endothelium, it reduced macrophages infiltration in media and adventitia. Furthermore, matrix metalloproteinase expression was markedly downregulated, accompanied with reduced expression of inflammatory mediators, including interleukin $1 \beta$, tumor necrosis factor- $\alpha$ and monocyte chemotactic protein-1. The present study revealed a novel target for the treatment of AAA and the anti-inflammatory effects of LMWF.
\end{abstract}

Correspondence to: Professor Zhenyu Shi, Department of Vascular Surgery, Zhongshan Hospital, Institute of Vascular Surgery, Fudan University, 180 Fenglin Road, Shanghai 200032, P.R. China E-mail: shizhenyumax@163.com

*Contributed equally

Key words: low molecular weight fucoidan, abdominal aortic aneurysm, P-selectin, leukocyte-endothelial cells interaction, inflammatory cytokines

\section{Introduction}

Abdominal aortic aneurysm (AAA) is characterized as a permanent dilation and degradation of the aortic wall. It is a life-threatening condition affecting $8.3 \%$ of the population over 65 years of age (1). Currently, there is no effective medication preventing or limiting the enlargement of AAA other than surgical intervention. Thus, it is important to investigate the mechanisms of AAA initiation and progression in order to develop new therapies. Through bioinformatics analysis, we found that focal adhesion was an important pathway in the development of AAA. Moreover, numerous basic researches and clinical studies have also reported that upregulation of adhesion molecules could promote the initiation of AAA through an inflammatory response induced by inflammatory cells infiltration (2-5).

P-selectin is an adhesion molecule localized to the surface of $\alpha$ granule in activated platelets and endothelial cells in human. It could bind to P-selectin ligand on leukocyte mediating the interaction between leukocytes with platelets or endothelial cells. In the elastase aortic perfusion model, P-selectin knockout could attenuate aneurysm formation (6). Previous study showed that Angiotensin (Ang) II could increase the expression of P-selectin on endothelia cells, which induced significant selectin-dependent leukocyte rolling, recruitment, and infiltration. Nevertheless, fucoidan could inhibit the leukocyte-endothelial cells interaction mediated by P-selectin (7). Low molecular weight fucoidan (LMWF) is a highly sulfated fraction degraded from fucoidan in Saccharina Japonica (8). It has been reported to present high affinity for P-selectin and abolish selectin-dependent recruitment of leukocytes (9). Thus, this study aimed to examine the effect of LMWF, as a competitive binding agent of P-selectin, in ApoE(-/-) mice on inflammatory infiltration and aneurysmal growth.

\section{Materials and methods}

Acquisition of microarray datasets. Two gene expression profiles (GSE47472 and GSE57691) were retrieved and downloaded from the National Center for Biotechnology Information GEO database (http://www.ncbi.nlm.nih.gov/geo). 
The dataset GSE47472, based on the platform of GPL10558 (Illumina HumanHT-12 V4.0 expression beadchip), consisted of 14 AAA neck samples from patients and 8 normal samples from donors following brain mortality. The array data of GSE57691, based on the same platform, included 20 AAA samples from patients with small AAA (mean maximum aortic diameter $=54.3 \pm 2.3 \mathrm{~mm}), 29$ AAA samples from patients with large AAA (mean maximum aortic diameter $=68.4 \pm 14.3 \mathrm{~mm}$ ) and 10 control aortic specimen of organ donors.

Data processing and enrichment analysis of DEGs. GEO2R (http://www.ncbi.nlm.nih.gov/geo/geo2r/) is an interactive tool that allows comparing two groups of samples in a GEO series. In this study, GEO2R was applied to screen differentially-expressed genes (DEGs) between AAA and normal aortic samples. The P-values were adjusted using Benjamini and Hochberg false discovery rate method. The threshold for the DEGs was set as adjusted P-value $<0.05$. Particularly, in GSE57691, the normal aortic group was compared with small AAA group and large AAA group, respectively. Venn plot was performed to determine the DEGs in all three datasets, which was then submitted to the Database for Annotation, Visualization and Integrated Discovery (DAVID; http://david.abcc.ncifcrf.gov/) for functional annotation analysis $(10,11)$. The significant enrichment analysis of DEGs was assessed based on Kyoto Encyclopedia of Genes and Genomes (KEGG; http://www.genome.jp/kegg/kegg2. html) with P-value $<0.05$ as the threshold.

Experimental design and AAA model. All experiments involving live animals were conducted in compliance with the 'Guide for the Care and Use of Laboratory Animals' and were approved by the institutional review board of Zhongshan Hospital, Fudan University (Shanghai, China). Six-month-old male apolipoprotein E-deficient (apoE-/-) mice weighing 35-40 g were obtained from Shanghai Lab. Animal Research Center. Ang II (1,000 ng/kg/min; Sigma-Aldrich; Merck KGaA, Darmstadt, Germany) or saline was administered subcutaneously for 28 days via Alzet osmotic minipumps (model 2004; DURECT, Cupertino, CA, USA) as described previously (12). Thirty-six mice were randomly allocated to 3 groups: Control, Ang II and LMWF group. LMWF group mice were given $200 \mathrm{mg} / \mathrm{kg} / \mathrm{d}$ LMWF (dissolved in $0.9 \%$ saline) by gavage for 35 days, which was started 1 week before minipump implantation. Identical volume of $0.9 \%$ saline were given to the other 2 groups for the same period by gavage. All these mice were fed with standard diet and water, and housed with a $12 \mathrm{~h}$ light/dark cycle. The preparation method of LMWF was detailed before (8).

Measurement of aortic size using ultrasonography. Aortic diameter measurements, intraluminal thrombus and intimal flap recognition were obtained via a high-frequency ultrasound system (Vevo 3100; VisualSonics, Toronto, Canada) in a blind manner for each group. The short axis view was applied to measure the maximum anterior-posterior diameter of abdominal aorta. The aortic size was measured before minipump implantation and 14 , and 28 day after. The AAA is defined as a $50 \%$ increase in maximum diameter of the abdominal aorta or the presence of abdominal aortic dissection. One experienced operators who were blind to the study design performed the quantitative analysis of the ultrasound imaging. In addition, mice were daily monitored for mortality analysis.

Peripheral blood monocytes count. After 4-weeks intervention, mice were anesthetized by intraperitoneal injection of $0.12-0.15 \mathrm{ml}$ sodium pentobarbital $(10 \mathrm{mg} / \mathrm{ml})$. Blood was collected in citrated tubes for complete blood count analysis. Peripheral blood monocytes were classified and quantified based on the size and granularity of cells, and content of nucleic acid using an automated hematology analyzer (Mindray BC-2800vet; Mindray Bio-Medical Electronics Co., Ltd., Shenzhen, China). Counts were expressed as $10^{9} / 1$ of blood.

Histologic studies and immuno-histochemical staining. Mice were sacrificed by cervical dislocation and perfused briefly with phosphate-buffered saline (PBS). Aortic segments were harvested and divided into two parts: one for biochemical analysis and one was fixed with $4 \%$ paraformaldehyde in PBS overnight. Then, it was embedded in paraffin and sectioned (5 $\mu \mathrm{m}$ in thickness). Hematoxylin and eosin (H\&E) staining and Elastin van Gieson (EVG) staining was used to show the gross structure of aorta and medial elastin, respectively. Immune-histochemical (IHC) assay was performed to identify P-selectin expression in endothelium, F4/80, and matrix metalloproteinases (MMP) expression in the aortic wall. In brief, sections were de-paraffinized, rehydrated, and subjected to heat-mediated antigen retrieval. After blocking with $3 \%$ bovine serum albumin for $30 \mathrm{~min}$ at room temperature, the sections were incubated with primary antibodies against P-selectin (1:100; sc-8419; Santa Cruz Biotechnology, Inc., Dallas, TX, USA), F4/80 (1:100; ab6640), MMP-2 (1:100; ab37150), MMP-9 (1:100; ab38898; Abcam, Cambridge, UK) overnight at $4^{\circ} \mathrm{C}$. Then, incubated with second antibody (1:500; Miaotong, Shanghai, China) for $50 \mathrm{~min}$ at room temperature. Histological sections were examined under a light microscope (BX53; Olympus Corp., Tokyo, Japan). Five random microscopic fields in each mouse ( $n=6$ in each group) were evaluated. Macrophage was expressed as number of F4/80-positive cells per square millimeter. P-selectin were quantified by the intensity and range of IHC staining in endothelium.

Reverse transcription-quantitative polymerase chain reaction $(R T-q P C R)$. Pro-inflammatory cytokines in the aortic tissues, including monocyte chemotactic protein-1 (MCP-1), tumor necrosis factor- $\alpha$ (TNF- $\alpha$ ), and interleukin $1 \beta$ (IL-1 $\beta$ ), were determined by qPCR. Total RNA was isolated using $1 \mathrm{ml}$ TRIzol Reagent (Takara Bio Inc., Kusatsu, Japan). Complementary DNA was prepared with $1 \mu \mathrm{g}$ total RNA using a Reverse Transcription kit (Takara Bio Inc.). Specific mRNAs were amplified using SYBR-Green PCR Mix (Takara Bio Inc.) in an ABI PRISM 7500 thermal cycler (Applied Biosystems; Thermo Fisher Scientific, Inc., Waltham, MA, USA). Cycling conditions were $95^{\circ} \mathrm{C}$ for $30 \mathrm{sec}$, followed by 40 cycles at $95^{\circ} \mathrm{C}$ for $5 \mathrm{sec}, 60^{\circ} \mathrm{C}$ for $34 \mathrm{sec}$. The primer sequences were as follows: MCP-1: forward, 5'-CTTCTGGGCCTGCTGTTCA-3' and reverse, 5'-CCAGCCTACTCATTGGGATCA-3'; TNF- $\alpha$ forward, 5'-CССTCACACTCAGATCATCTTCT-3' and reverse, 5'-GCTACGACGTGGGCTACAG-3'; IL-1 $\beta$ forward, 
A

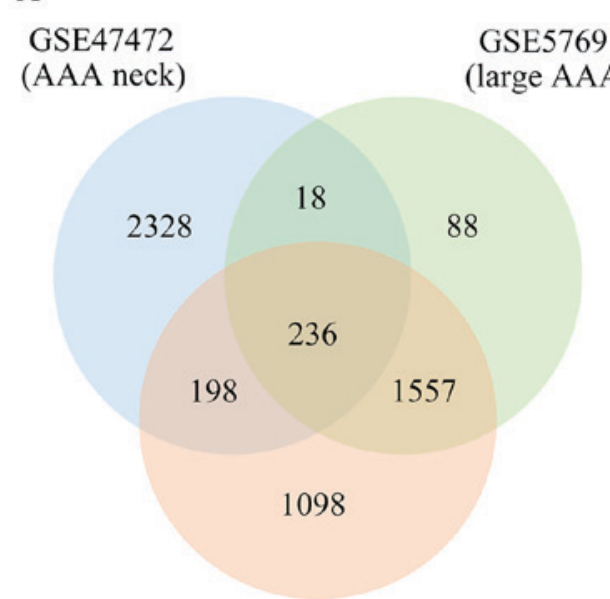

GSE57691 (small AAA)
B

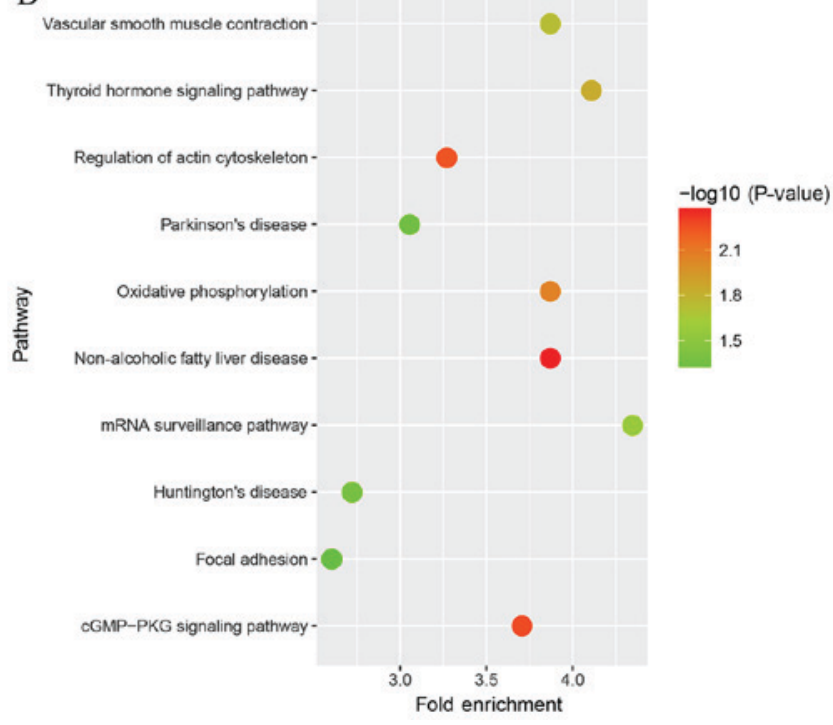

Figure 1. Bioinformatic analysis: (A) Identification of common differentially expressed genes (DEGs) in mRNA expression profiles; (B) Kyoto Encyclopedia of Genes and Genomes enrichment scatter plot of DEGs. The y-axis shows the name of the pathway, and the x-axis represents the Fold Enrichment. The dot color indicates the-log 10 (P-value).

5'-GCAACTGTTCCTGAACTCAACT-3' and reverse, 5'-ATC TTTTGGGGTCCGTCAACT-3'; $\beta$-actin forward, 5'-CGA GGCCCAGAGCAAGAGAGGTAT-3' and reverse, 5'-CAC GGTTGGCCTTAGGGTTCA-3'; The relative expression levels of mRNA were calculated with $\beta$-actin mRNA as the invariant control using the $2^{-\Delta \Delta C q}$ formula (13).

Western blotting. Aortic tissues were lysed by RIPA buffer. The protein concentration was measured using a BCA protein Assay kit. Then, $50 \mu \mathrm{g}$ total proteins were separated by $10 \%$ SDS-PAGE and transferred onto $0.45 \mu \mathrm{m}$ PVDF transfer membranes (EMD Millipore, Billerica, MA, USA). After blocked with $5 \%$ non-fat milk for $1 \mathrm{~h}$ at room temperature, the membranes were incubation with primary antibodies at $4^{\circ} \mathrm{C}$ overnight, including MMP2 (1:1,000; ab37150), MMP9 (1:1,000; ab38898; both Abcam), $\beta$-actin $(1: 1,000$; BS6007M; Bioworld Technology, Inc., St. Louis Park, MN, USA). Then the membranes were washed and incubated with secondary antibody (1:3,000, Bioworld) for $30 \mathrm{~min}$ at room temperature and detected using an enhanced chemiluminescence system. The bands relative intensities were analyzed using AlphaEase FC software (USA).

Statistical analysis. Results are expressed as means \pm standard deviations or proportions. Comparisons between groups were performed by one-way ANOVA analysis followed by a post hoc Bonferroni test for intergroup pairwise comparisons (Prism 5; GraphPad Software Inc., San Diego, USA). For the incidence difference of AAA and intraluminal thrombus between Ang II group and LMWF group, a Fisher's exact test was applied (Stata 12.0; StataCorp, College Station, TX, USA). P<0.05 was considered to indicate a statistically significant difference.

\section{Results}

Focal adhesion is involved in the initiate and development of $A A A$. A total of 2,780, 1,899 and 3,089 genes were identified as the DEGs from these datasets, respectively. Among these DEGs, 236 genes were found in all three datasets (Fig. 1). Through pathway enrichment analysis, these DEGs were identified to be significantly enriched in non-alcoholic fatty liver disease, cGMP-PKG signaling pathway, regulation of actin cytoskeleton, oxidative phosphorylation, thyroid hormone signaling pathway, vascular smooth muscle contraction, mRNA surveillance pathway, Huntington's disease, Parkinson's disease and focal adhesion (Fig. 1).

LMWF limits the enlargement of experimental AAA but not the incidence. All experimental AAA induced by Ang II infusion developed above and around renal arteries. There were no significant differences in body weight among the 3 groups before and after the intervention. One mouse died from abdominal aortic rupture 18 day after minipump implantation in the Ang II group. All mice survived till the end of intervention in the other 2 groups. The incidence of AAA formation was not significantly different between LMWF group and Ang II group (LMWF group, 33\%; Ang II group, $75 \% ; \mathrm{P}=0.100)$. However, LMWF administration significantly decreased the maximal aortic diameter (Control group, 0.84 $\pm 0.06 \mathrm{~mm}$; LMWF group, $1.30 \pm 0.27 \mathrm{~mm}$; Ang II group, $1.63 \pm 0.45 \mathrm{~mm}$ ) (Fig. 2). As to the histological changes of AAA formation, H\&E staining showed unusual medial thickening and infiltration of inflammatory cells. EVG staining revealed the degeneration and destruction of the medial elastic layers. However, LMWF administration prevented the thickening of medial and degeneration of the aortic wall (Fig. 3).

Circulating monocytes, intraluminal thrombus and P-selectin are not suppressed by LMWF administration. Complete blood count analysis showed that peripheral blood monocytes were not significantly different in blood samples among the three groups. Compared with Ang II group, the incidence of intraluminal thrombus was also not decreased by LMWF administration (LMWF group, 41.7\%; Ang II group, 54.5\%; 

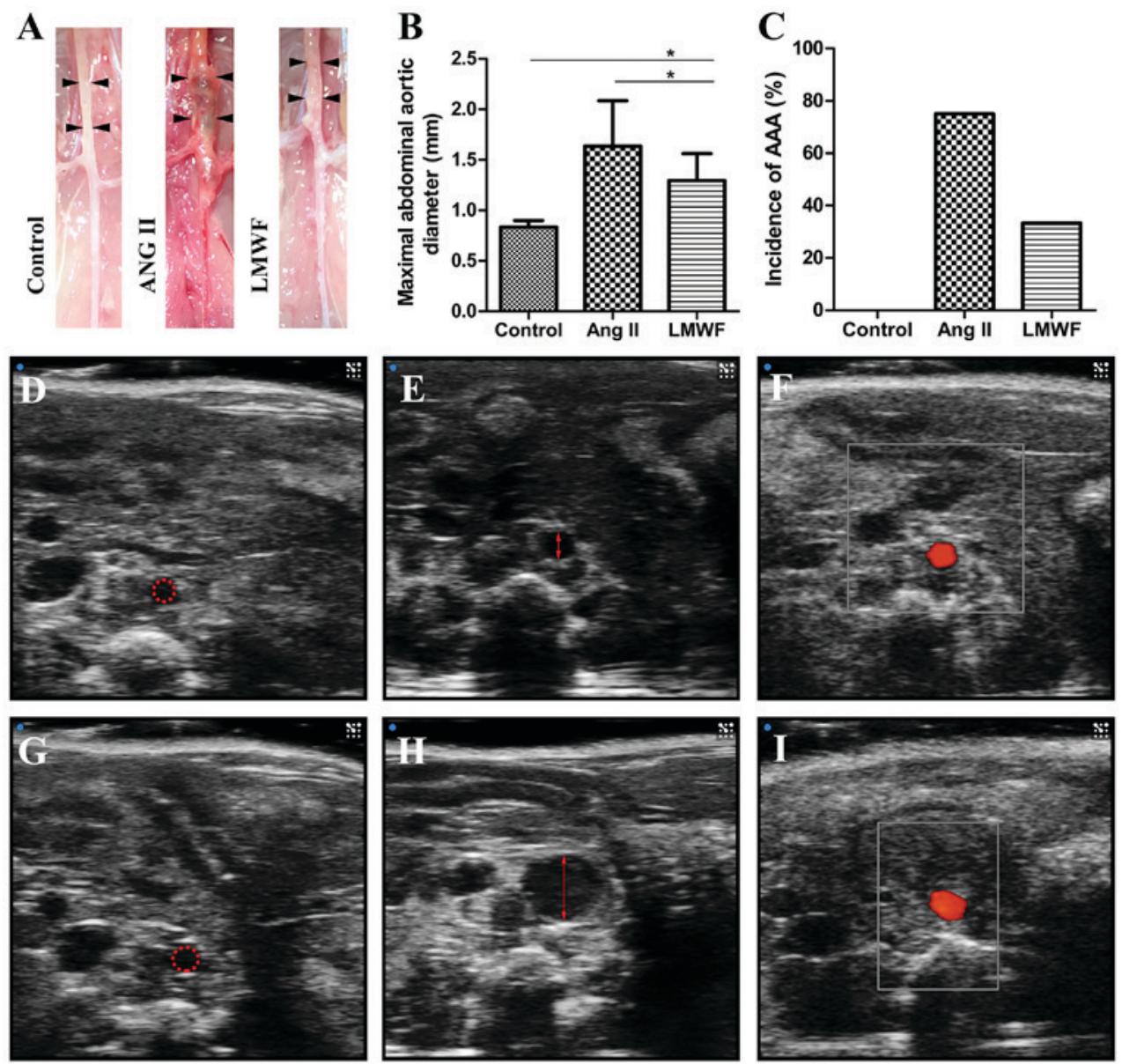

Figure 2. (A) Representative images of abdominal arteries after 4-weeks intervention; Black arrows show the left and right sides of the abdominal aorta. (B) Bar graph of maximal abdominal aortic diameter $(" \mathrm{P}<0.05)$; (C) Graphic depiction of the incidence of abdominal aortic aneurysm. Representative ultrasonography images of abdominal arteries of Control (D), Ang II (E), LMWF group (F) at baseline. Representative ultrasonography images of maximal abdominal aortic diameter of Control (G), Ang II (H), LMWF group (I) after 4-weeks intervention. Red highlighting indicates the lumen of abdominal aorta. AAA, abdominal aortic aneurysm; Ang, Angiotensin; LMWF, low molecular weight fucoidan.
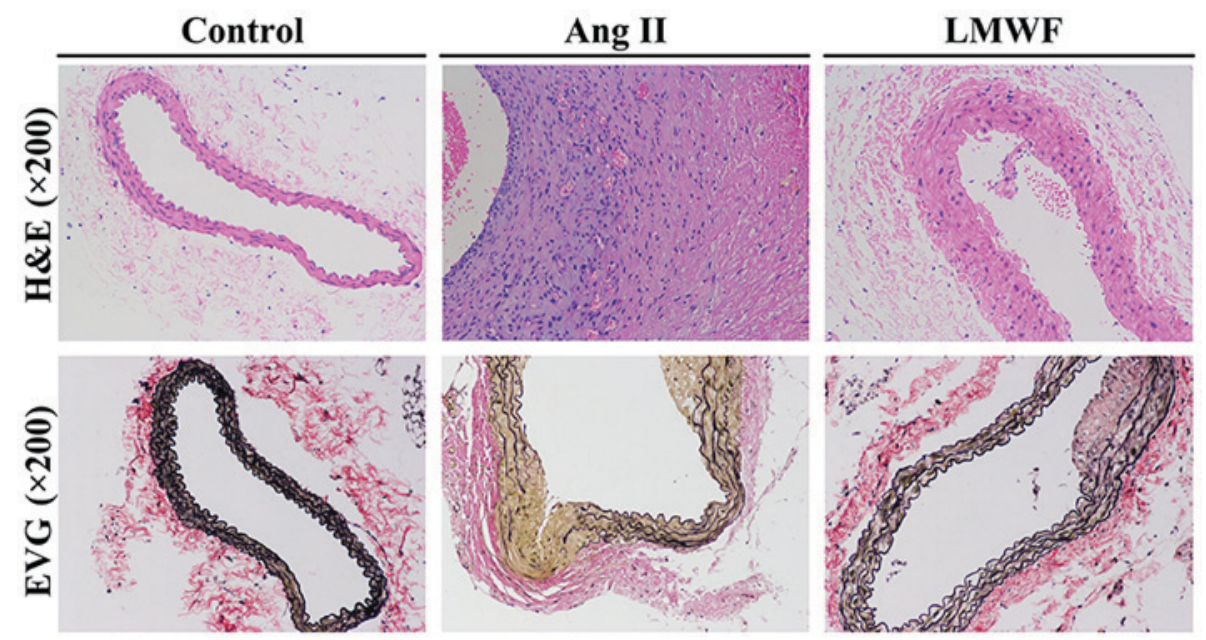

Figure 3. Histological analysis (hematoxylin and eosin staining and Elastin van Gieson staining) of the aorta. Original magnification, x200. Ang, Angiotensin; LMWF, low molecular weight fucoidan.

$\mathrm{P}=0.414)$. In addition, the upregulation of $\mathrm{P}$-selectin in aortic intima was found in Ang II group. However, this effect of Ang II on P-selectin expression was independent of LMWF administration (Fig. 4).
LMWF inhibits inflammatory infiltration. In the Ang II group, an abundance of invasive macrophages was observed in the aortic wall. The number of F4/80-positive cells were significantly lower in the LMWF group than the Ang II group. 
A
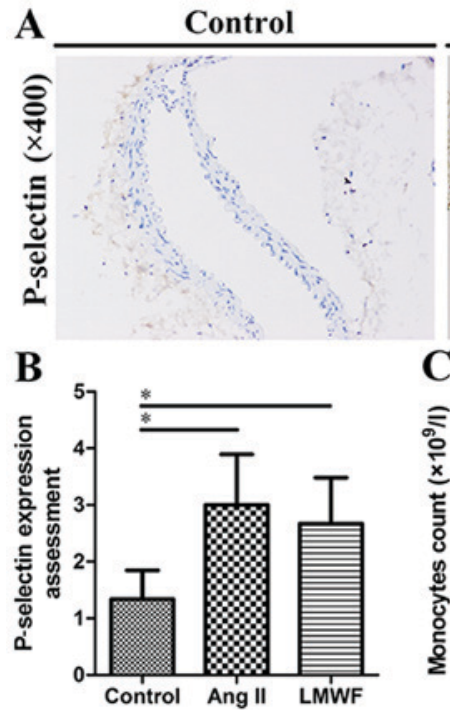

Ang II
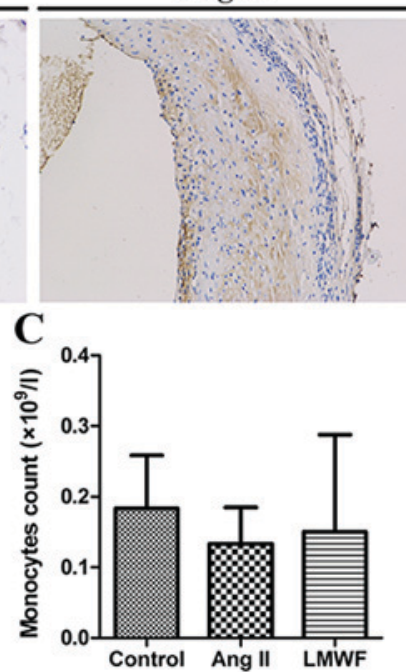

LMWF

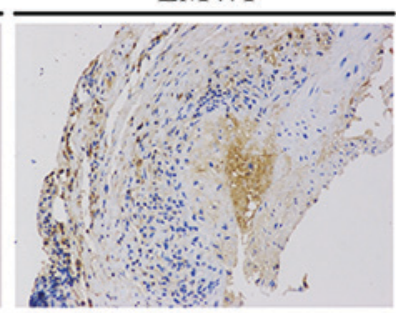

D

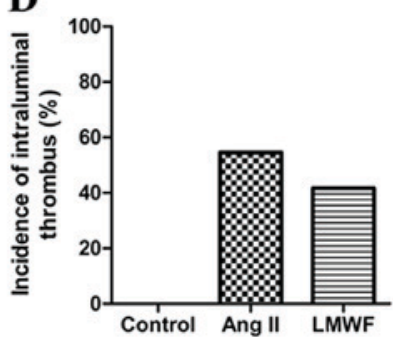

Figure 4. (A) Representative images of immune-histochemical stains for P-selectin. Original magnification, x400; (B) Quantification of P-selectin shown as the intensity and range of staining in endothelium ( $\left.{ }^{*}<0.05\right)$; (C) Bar graph of circulatory monocytes count; (D) Graphic depiction of the incidence of intraluminal thrombus. Ang, Angiotensin; LMWF, low molecular weight fucoidan.

Moreover, the expression of TNF- $\alpha$, MCP-1 and IL-1 $\beta$ were significantly higher in the Ang II group than in the Control group. LMWF significantly decreased TNF- $\alpha$, MCP-1 and IL-1 $\beta$ expression induced by inflammatory cells infiltration. Besides, IHC and Western Blot assay of AAA tissues demonstrated that MMP expression was enhanced in AAA tissues than that in Control group. MMP-2 and MMP-9 expressions were significantly lower in LMWF group than that in Ang II group (Fig. 5).

\section{Discussion}

It is well documented that the pathogenesis of AAA is complicated and multifactorial. In this study, we attempted to detect the potential mechanism by bioinformatic analysis. Based on the microarray data contributed by Biros et al, we identified 236 common DEGs and 10 pathways, which may affect the development of AAA $(14,15)$. Notably, the tissue used in the microarray analysis included AAA neck, small-AAA aortic wall and large-AAA aortic wall, which indicated the formation and development of AAA. Although the fold enrichment of focal adhesion was relatively low, it still suggested the importance of leukocytes adhering to endothelium except for apoptosis of vascular smooth muscle cells and degradation of the extracellular matrix in the development of AAA. Thus, we aimed to detect the possibility of limiting the enlargement of AAA by interfering leukocyte-endothelial interactions.

$\mathrm{P}$-selectin is an adhesion molecule expressed on the endothelial cells. In vitro, P-selectin expression in endothelial cells and macrophage adhesion were increased by Ang II, which could be inhibited by fucoidan (7). Besides, accumulating evidence has determined that medial and adventitial macrophage activity promotes aneurysmal degeneration via production of MMP, pro-inflammatory cytokines, and reactive oxygen species $(16,17)$. In this study, we found circulating monocytes was not changed by LMWF administration, as well as P-selectin expressed in the endothelial cells of AAA.
However, the invasive macrophages were significantly lower in the medial of abdominal aorta. PCR and IHC analysis also confirmed that inflammatory mediators and MMP expressions were also significantly decreased in AAA tissue. These results concluded that dietary intake of LMWF could limit the enlargement of AAA in Ang II-infused ApoE-/-mice, at least in part, by blocking the adhesion site of macrophages and endothelium, and thus decreasing the inflammatory mediators, MMP-2 and MMP-9 production from macrophage infiltration. Furthermore, LMWF was also proved the potential to directly modulate the expression of MMP-2 (18).

In a previous study, Alsac et al referred that neutrophil rolling and trapping in the intraluminal thrombus were mainly mediated by $\mathrm{P}$-selectin and P-selectin glycoprotein ligand-1 on the activated platelets and neutrophil surface, which led to the death and releasing proteolytic enzymes of neutrophil (19). Thus, they detected the effect of fucoidan on leukocyte-thrombus interaction in an AAA model, which was characterized by neutrophil-rich intraluminal thrombus induced by repeated episodes of weak bacteremia. Finally, they found that fucoidan could limit the enlargement of AAA by decreasing neutrophil activation. However, the AAA model used in our study was different from theirs. It was induced by traditional cardiovascular risk factors, including atherosclerotic lesions and hypertension. The incidence of intraluminal thrombus and P-selectin expression in endothelial cells were not significantly different between Ang II group and LMWF group. Moreover, circulating monocytes were not significantly different among these groups. Nevertheless, the invasive macrophages in the media were significantly lower by LMWF through interfering the leukocyte-endothelial cells interaction. Thus, the mechanism we clarified was a supplement to theirs based on a different animal model, which also indicated that both endothelial cell and platelet P-selectin contributed to the formation of AAA.

In fact, increased infiltration of inflammatory cells was an established pathological hallmark of AAA. During the initiate 
A
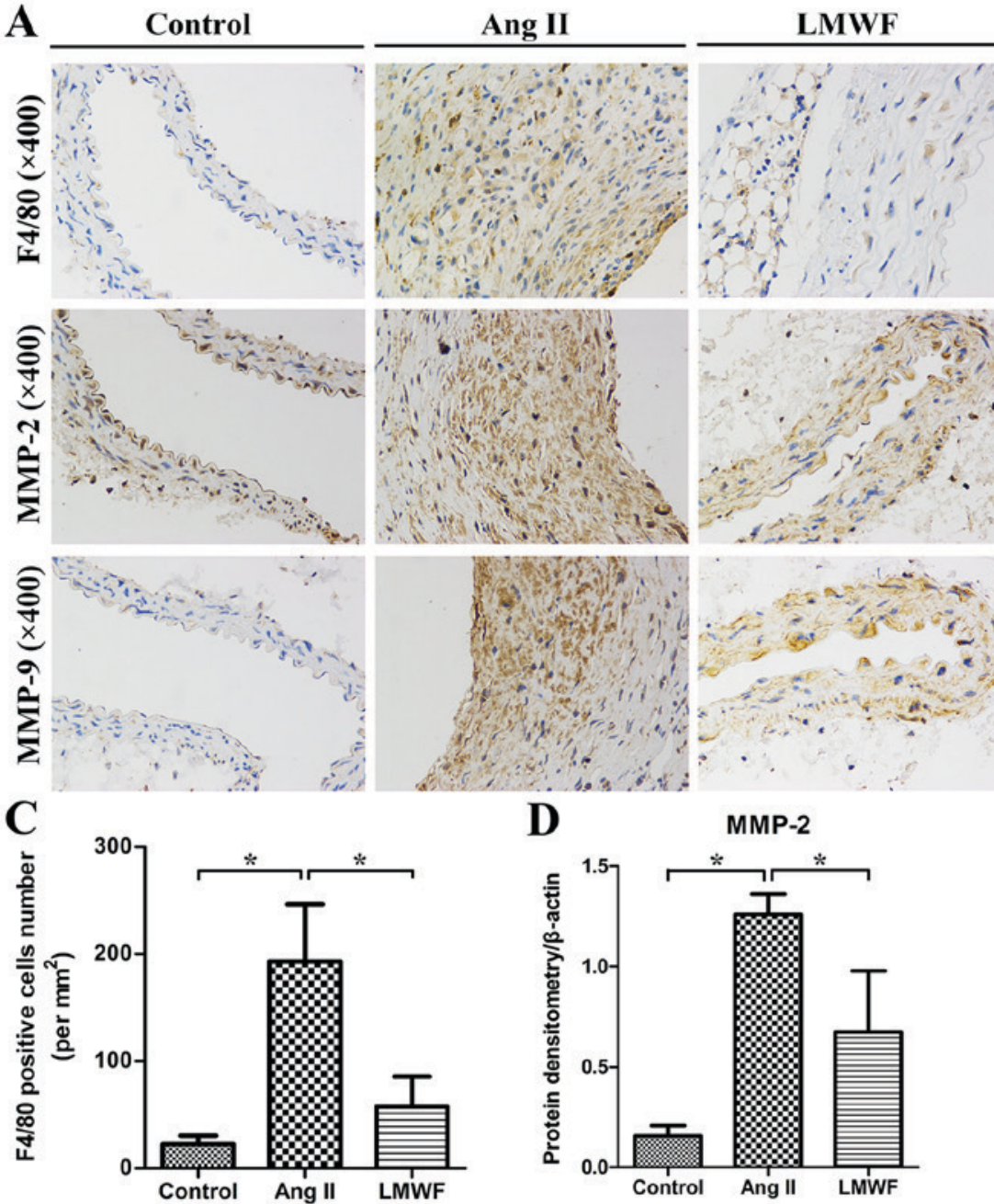

$\mathbf{F}$

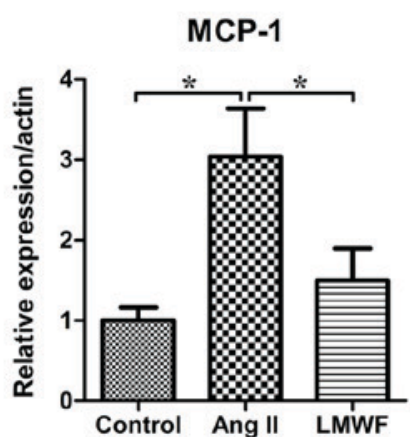

D
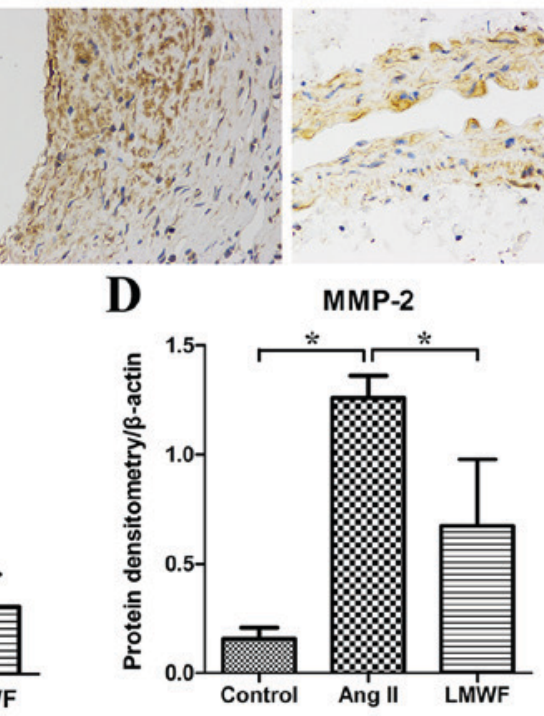

G

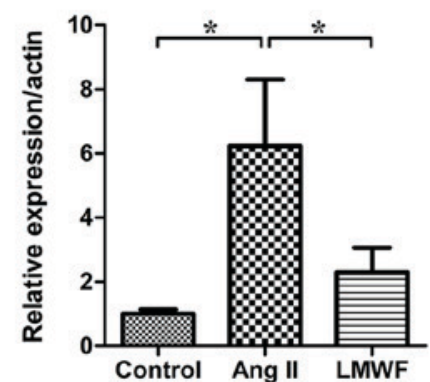

B

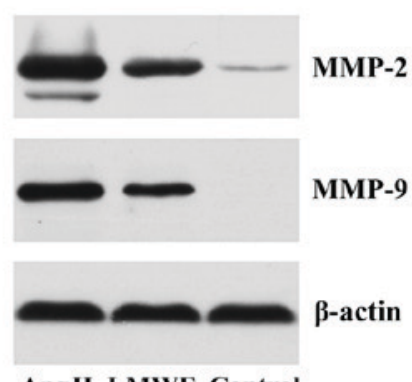

Ang II LMWF Control
$\mathbf{E}$

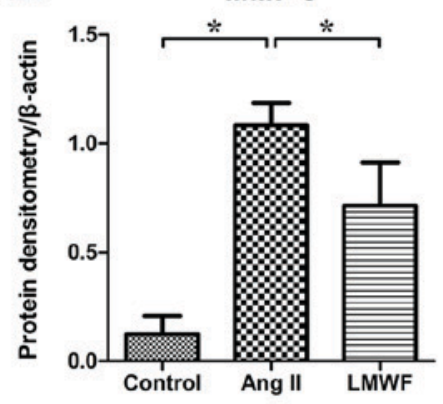

H

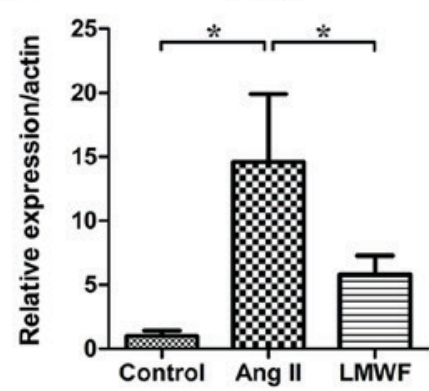

Figure 5. (A) Representative images of immune-histochemical stains for F4/80, MMP-2 and MMP-9. Original magnification, x400; (B) Western blot results of MMP-2 and MMP-9 in abdominal arteries; (C) Quantification of macrophage infiltration, shown as the number of positive cells per square millimeter $\left({ }^{*} \mathrm{P}<0.05\right)$. Quantitative analysis of MMP-2 (D) and MMP-9 (E) expression $\left({ }^{*} \mathrm{P}<0.05\right)$. mRNA expression of MCP-1 (F), TNF- $\alpha(\mathrm{G})$ and IL-1 $\beta$ (H) $\left({ }^{*} \mathrm{P}<0.05\right)$. MMP, metalloproteinases; MCP, monocyte chemotactic protein.

of inflammatory cells migrating into sub-endothelial space, focal adhesion plays an important role. Previously, numerous efforts have been made to interfere the leukocyte-endothelial cell interaction $(20,21)$. Considering that Ang II could induce endothelial activation and dysfunction, calcium channel inhibitors and Ang II receptor antagonists have been proved to prevent the Ang II-induced over-expression of adhesion molecules and chemokines, and presented a good endothelial protective activity $(22,23)$. Another important mechanism was the monocyte chemoattraction induced by Ang II, leukocyte-endothelial cell interaction could be inhibited by anti-chemokines without affecting the endothelial expression of P-selectin and VCAM-1 (24). Iida et al also detected that peptide inhibitor of CXCL4-CCL5 heterodimer formation could attenuate AAA in mice due to that it could inhibit CCL5-mediated monocyte chemotaxis and arrest on activated endothelial cells in vitro (25). In this study, the decreased inflammatory mediators also confirm the importance of chemokines.

The adhesion character of fucoidan was also applied in the imaging of AAA. In some studies, P-selectin was described as 
a molecular imaging target for AAA early detection, dilatation, and rupture risk assessment $(26,27)$. A team from France developed microcapsules functionalized with fucoidan to target P-selectin, enabling detection of activated endothelium and thrombosis of AAA in vivo (28). Notably, LMWF is highly sulfated fractions degraded from fucoidan, with higher affinity for P-selectin. The structure property of fucoidan was associated with its specific biological activity. LMWF was more potent than fucoidan in endothelial protection (29). Moreover, LMWF could induce endothelial cell migration via the PI3K/AKT pathway and promote angiogenesis in a rat model of critical hindlimb ischemia (30). As to the character of binding to activated human platelets, LMWF could suppress the thrombin-induced platelet aggregation and has a potential to become an oral antithrombotic agent (31-33). In addition, Deux et al found that LMWF prevented neointimal hyperplasia in rabbit iliac artery in-stent restenosis model (34). Recently, LMWF has been used in food supplements and pharmaceutical products (35). Toxicological evaluation of LMWF in vitro and in vivo have verified that there were no toxicological effects at a high dose (36). Overall, LMWF showed a promising future in the detection and treatment of vascular diseases.

Our study has several limitations. First, although the incidence of ILT was not significantly different between the two groups, the volume of ILT was not quantified. We still could not exclude the potential effect of activated platelet and thrombosis. Second, a single dose of LMWF was applied in this study. Third, macrophages also produce pro-inflammatory cytokines, which we could not identify whether LMWF could directly reduce these chemokines. Fourth, MMPs activities were not tested in the present study, which was suggested for future studies. Last, the expression of P-selectin in the endothelial cells could not be quantified by western blot analysis. The single section of IHC analysis may be unstable.

In conclusion, focal adhesion involved in the initiate and development of AAA. Dietary intake of LMWF limited AAA enlargement through inhibiting P-selectin-dependent leukocytes roll on the endothelium to initiate leukocyte recruitment, adhesion and infiltration. These results suggest that LMWF may have beneficial effects against AAA enlargement.

\section{Acknowledgements}

Not applicable.

\section{Funding}

The present study was supported by the National Natural Science Foundation of China (grant no. 81370424).

\section{Availability of data and materials}

The datasets used and analyzed during the current study are available from the corresponding author on reasonable request.

\section{Authors' contributions}

ZS and CL contributed to the conception and design; LC and YW performed the data collection and interpretation; $\mathrm{MZ}$ and YD analyzed the datasets and wrote this article. ZS obtained the funding. All authors read and approved the final manuscript.

\section{Ethics approval and consent to participate}

All experiments involving live animals were conducted in compliance with the 'Guide for the Care and Use of Laboratory Animals' and approved by the Institutional Review Board of Zhongshan Hospital, Fudan University.

\section{Consent for publication}

Not applicable.

\section{Competing interests}

The authors declare that they have no competing interests.

\section{References}

1. Sampson UK, Norman PE, Fowkes FG, Aboyans V, Song Y, Harrell FE Jr, Forouzanfar MH, Naghavi M, Denenberg JO, McDermott MM, et al: Estimation of global and regional incidence and prevalence of abdominal aortic aneurysms 1990 to 2010. Global heart 9: 159-170, 2014.

2. Thomas M, Gavrila D, McCormick ML, Miller FJ Jr, Daugherty A, Cassis LA, Dellsperger KC and Weintraub NL: Deletion of p47phox attenuates angiotensin II-induced abdominal aortic aneurysm formation in apolipoprotein E-deficient mice. Circulation 114: 404-413, 2006.

3. Sharma AK, Lu G, Jester A, Johnston WF, Zhao Y, Hajzus VA, Saadatzadeh MR, Su G, Bhamidipati CM, Mehta GS, et al: Experimental abdominal aortic aneurysm formation is mediated by IL-17 and attenuated by mesenchymal stem cell treatment. Circulation 126 (11 Suppl 1): S38-S45, 2012.

4. Harrison SC, Smith AJ, Jones GT, Swerdlow DI, Rampuri R, Bown MJ; Aneurysm Consortium, Folkersen L, Baas AF, de Borst GJ, et al: Interleukin-6 receptor pathways in abdominal aortic aneurysm. Eur Heart J 34: 3707-3716, 2013.

5. Li MW, Mian MO, Barhoumi T, Rehman A, Mann K, Paradis P and Schiffrin EL: Endothelin-1 overexpression exacerbates atherosclerosis and induces aortic aneurysms in apolipoprotein E knockout mice. Arterioscler Thromb Vasc Biol 33: 2306-2315, 2013.

6. Hannawa KK, Cho BS, Sinha I, Roelofs KJ, Myers DD, Wakefield TJ, Stanley JC, Henke PK and Upchurch GR Jr: Attenuation of experimental aortic aneurysm formation in P-selectin knockout mice. Ann N Y Acad Sci 1085: 353-359, 2006.

7. Piqueras L, Kubes P, Alvarez A, O'Connor E, Issekutz AC, Esplugues JV and Sanz MJ: Angiotensin II induces leukocyte-endothelial cell interactions in vivo via AT(1) and AT(2) receptor-mediated P-selectin upregulation. Circulation 102: 2118-2123, 2000 .

8. Xu Y, Zhang Q, Luo D, Wang J and Duan D: Low molecular weight fucoidan modulates P-selectin and alleviates diabetic nephropathy. Int J Biol Macromol 91: 233-240, 2016.

9. Jin W, Wang J, Jiang H, Song N, Zhang W and Zhang Q: The neuroprotective activities of heteropolysaccharides extracted from Saccharina Japonica. Carbohydr Polym 97: 116-120, 2013.

10. Huang da W, Sherman BT and Lempicki RA: Systematic and integrative analysis of large gene lists using DAVID bioinformatics resources. Nat Protoc 4: 44-57, 2009.

11. Huang da W, Sherman BT and Lempicki RA: Bioinformatics enrichment tools: Paths toward the comprehensive functional analysis of large gene lists. Nucleic Acids Res 37: 1-13S, 2009.

12. Daugherty A and Cassis LA: Mouse models of abdominal aortic aneurysms. Arterioscler Thromb Vasc Biol 24: 429-434, 2004.

13. Livak KJ and Schmittgen TD: Analysis of relative gene expression data using real-time quantitative PCR and the 2(-Delta Delta C(T)) method. Methods 25: 402-408, 2001. 
14. Biros E, Moran CS, Rush CM, Gäbel G, Schreurs C,Lindeman JH, Walker PJ, Nataatmadja M, West M, Holdt LM, et al: Differential gene expression in the proximal neck of human abdominal aortic aneurysm. Atherosclerosis 233: 211-218, 2014.

15. Biros E, Gäbel G, Moran CS, Schreurs C,Lindeman JH, Walker PJ, Nataatmadja M, West M, Holdt LM, Hinterseher I, et al: Differential gene expression in human abdominal aortic aneurysm and aortic occlusive disease. Oncotarget 6: 12984-12996, 2015.

16. Yoshida S, Fuster JJ and Walsh K: Adiponectin attenuates abdominal aortic aneurysm formation in hyperlipidemic mice. Atherosclerosis 235: 339-346, 2014.

17. Lu HY, Huang CY, Shih CM, Chang WH, Tsai CS, Lin FY and Shih CC: Dipeptidyl peptidase-4 inhibitor decreases abdominal aortic aneurysm formation through GLP-1-dependent monocytic activity in mice. PLoS One 10: e0121077, 2015.

18. Hlawaty H, Suffee N, Sutton A, Oudar O, Haddad O, Ollivier V, Laguillier-Morizot C, Gattegno L, Letourneur D and Charnaux N: Low molecular weight fucoidan prevents intimal hyperplasia in rat injured thoracic aorta through the modulation of matrix metalloproteinase-2 expression. Biochem Pharmacol 81: 233-243, 2011.

19. Alsac JM, Delbosc S, Rouer M, Journé C, Louedec L, Meilhac O and Michel JB: Fucoidan interferes with Porphyromonas gingivalis-induced aneurysm enlargement by decreasing neutrophil activation. J Vasc Surg 57: 796-805, 2013.

20. da Cunha V, Tham DM, Martin-McNulty B, Deng G, Ho JJ, Wilson DW, Rutledge JC, Vergona R, Sullivan ME and Wang YX: Enalapril attenuates angiotensin II-induced atherosclerosis and vascular inflammation. Atherosclerosis 178: 9-17, 2005.

21. Cui W, Zheng Y, Zhang Q, Wang J, Wang L, Yang W, Guo C, Gao W, Wang X and Luo D: Low-molecular-weight fucoidan protects endothelial function and ameliorates basal hypertension in diabetic Goto-Kakizaki rats. Lab Invest 94: 382-393, 2014.

22. Iida Y, Xu B, Schultz GM, Chow V, White JJ, Sulaimon S, Hezi-Yamit A, Peterson SR and Dalman RL: Efficacy and mechanism of angiotensin II receptor blocker treatment in experimental abdominal aortic aneurysms. PLoS One 7: e49642, 2012.

23. Mieth A, Revermann M, Babelova A, Weigert A, Schermuly RT and Brandes RP: L-type calcium channel inhibitor diltiazem prevents aneurysm formation by blood pressure-independent anti-inflammatory effects. Hypertension 62: 1098-1104, 2013.

24. Mateo T, Abu Nabah YN, Abu Taha M, Mata M,Cerdá-Nicolás M, Proudfoot AE, Stahl RA, Issekutz AC, Cortijo J, Morcillo EJ, et al: Angiotensin II-induced mononuclear leukocyte interactions with arteriolar and venular endothelium are mediated by the release of different CC chemokines. J Immunol 176: 5577-5586, 2006.

25. Iida Y, Xu B, Xuan H, Glover KJ, Tanaka H, Hu X, Fujimura N, Wang W, Schultz JR, Turner CR and Dalman RL: Peptide inhibitor of CXCL4-CCL5 heterodimer formation, MKEY, inhibits experimental aortic aneurysm initiation and progression. Arterioscler Thromb Vasc Biol 33: 718-726, 2013.
26. Li B, Juenet M, Aid-Launais R, Maire M, Ollivier V,Letourneur D and Chauvierre C: Development of polymer microcapsules functionalized with fucoidan to target P-selectin overexpressed in cardiovascular diseases. Adv Healthc Mater 6, 2017.

27. Bonnard T, Serfaty JM, Journé C, Ho Tin Noe B, Arnaud D, Louedec L, Derkaoui SM, Letourneur D, Chauvierre C and Le Visage C: Leukocyte mimetic polysaccharide microparticles tracked in vivo on activated endothelium and in abdominal aortic aneurysm. Acta Biomater 10: 3535-3545, 2014.

28. Rouzet F, Bachelet-Violette L, Alsac JM, Suzuki M, Meulemans A, Louedec L, Petiet A, Jandrot-Perrus M, Chaubet F and Michel JB: Radiolabeled fucoidan as a p-selectin targeting agent for in vivo imaging of platelet-rich thrombus and endothelial activation. J Nucl Med 52: 1433-1440, 2011.

29. Chen A, Lan Y, Liu J, Zhang F, Zhang L, Li B and Zhao X: The structure property and endothelial protective activity of fucoidan from Laminaria japonica. Int J Biol Macromol 105: 1421-1429, 2017.

30. Bouvard C, Galy-Fauroux I, Grelac F, Carpentier W, Lokajczyk A, Gandrille S, Colliec-Jouault S, Fischer AM and Helley D: Low-molecular-weight fucoidan induces endothelial cell migration via the PI3K/AKT pathway and modulates the transcription of genes involved in angiogenesis. Mar Drugs 13: 7446-7462, 2015 .

31. Zhu Z, Zhang Q, Chen L, Ren S, Xu P, Tang Y and Luo D: Higher specificity of the activity of low molecular weight fucoidan for thrombin-induced platelet aggregation. Thromb Res 125: 419-426, 2010.

32. Bachelet L, Bertholon I, Lavigne D, Vassy R, Jandrot-Perrus M, Chaubet F and Letourneur D: Affinity of low molecular weight fucoidan for P-selectin triggers its binding to activated human platelets. Biochim Biophys Acta 1790: 141-146, 2009.

33. Zhao X, Guo F, Hu J, Zhang L, Xue C, Zhang Z and Li B: Antithrombotic activity of oral administered low molecular weight fucoidan from Laminaria Japonica. Thromb Res 144: 46-52, 2016.

34. Deux JF, Meddahi-Pellé A, Le Blanche AF, Feldman LJ, Colliec-Jouault S, Brée F, Boudghène $F$, Michel JB and Letourneur D: Low molecular weight fucoidan prevents neointimal hyperplasia in rabbit iliac artery in-stent restenosis model. Arterioscler Thromb Vasc Biol 22: 1604-1609, 2002.

35. Tsai HL, Tai CJ, Huang CW, Chang FR and Wang JY: Efficacy of low-molecular-weight fucoidan as a supplemental therapy in metastatic colorectal cancer patients: A double-blind randomized controlled trial. Mar Drugs 15: pii: E122, 2017.

36. Hwang PA, Yan MD, Lin HT, Li KL and Lin YC: Toxicological evaluation of low molecular weight fucoidan in vitro and in vivo. Mar Drugs 14: pii: E121, 2016.

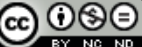

This work is licensed under a Creative Commons

Attribution-NonCommercial-NoDerivatives 4.0 International (CC BY-NC-ND 4.0) License. 